\title{
QUADRATURE FORMULAS ON THE UNIT CIRCLE AND TWO-POINT PADÉ APPROXIMATION
}

\author{
ADHEMAR BULTHEEL \\ Dept. Computer Science, K.U. Leuven, Belgium \\ PABLO GONZÁLEZ-VERA \\ Dept. Mathematical Analysis, Univ. of La Laguna, Tenerife, Spain \\ ERIK HENDRIKSEN \\ Dept. Mathematics, Univ. of Amsterdam, The Netherlands \\ and \\ OLAV NJÅSTAD \\ Dept. Mathematical Sciences, Univ. of Trontheim-NTH, Norway
}

\begin{abstract}
In this paper our aim is to estimate integrals of the form $I_{\mu}\{f\}=\int_{-\pi}^{\pi} f\left(e^{i \theta}\right) d \mu(\theta)$ where $\mu$ is, in general a complex measure. We consider quadrature formulas like $I_{n}\{f\}=\sum_{j=1}^{n} A_{j, n} f\left(x_{j, n}\right)$ with $n$ distinct nodes $x_{j, n}$ on the unit circle and so that $I_{\mu}\{f\}=I_{n}\{f\}$ for any $f \in \mathcal{R}_{n}$ (a certain subspace of Laurent polynomials with dimension $n$ ). Under appropriate assumptions on the function $f$ we show that such quadratures are immediately related to the estimation of the so-called Riesz-Herglotz transform of the measure $\mu$. Results concerning the rate of convergence of these quadratures are also given.
\end{abstract}

Key words: Numerical quadrature, two-point Padé approximation

\section{Introduction}

In the approximate calculation of definite integrals,

$$
\int_{a}^{b} f(x) \omega(x) d x
$$

where $[a, b]$ is a finite interval and $\omega$ a complex function, by a linear quadrature formule with coefficients or weights $A_{j}$ and nodes $x_{j}, j=1,2, \ldots, n$, it is fundamental that $f$ can be uniformly approximated by polynomials on $[a, b]$. Thus the coefficients and nodes will be chosen so that the corresponding quadrature $\sum_{j=1}^{n} A_{j} f\left(x_{j}\right)$ integrates exactly polynomials up to a certain degree as large as possible. When $\omega(x) \geq 0$, the well known Gauss-Christoffel formulas arise. See e.g. [13] for a survey. When $\omega$ is in general a complex function, not so many results are known. Orthogonal polynomials with respect to complex measures play now a crucial role. A recent contribution in this matter is the paper by González-Vera et al [16]. (See also, [25] and $[1])$.

On the other hand, the estimation of integrals not in the form (1) but now on 
the unit circle, that is

$$
I_{\mu}\{f\}=\int_{-\pi}^{\pi} f\left(e^{i \theta}\right) d \mu(\theta)
$$

and related topics such as Szegö polynomials and the trigonometric moment problem have recently received much attention as a result of their application in the rapidly growing field of digital signal processing $([3,11])$ and in operator theory $[15,22]$. In such cases $\mu$ in (2) used to be a finite positive Borel measure on $[-\pi, \pi]$ and the concept of "Szegö quadrature formula" appears as an anologue on the unit circle to a Gaussian qaudrature formula on an interval. It was formally introduced by W.B. Jones, O. Njåstad and W. Thron in [21], (see also [26]). Results concerning the error and convergence for such formulas were given in [5]. As for numerical results see [18].

Finally, the extension of these quadratures to certain spaces of rational functions with prescribed poles not on the unit circle were first introduced in [4]. (See also $[6,7]$ with an application to the Poisson integral).

Next, some remarks on notation are given. For every pair $(p, q)$ of integers, where $p \leq q$, we denote by $\Delta_{p, q}$ the linear space of all Laurent polynomials ( $L$-polynomials)

$$
L(z)=\sum_{j=p}^{q} c_{j} z^{j} ; \quad c_{j} \in \mathbf{C} .
$$

We write $\Delta$ for the linear space of all $L$-polynomials, $\Pi$ for the space of all polynomials and $\Pi_{n}\left(=\Delta_{0, n}\right)$ for the space of all polynomials of degree at most $n$. We shall also write $\mathbf{T}=\{w \in \mathbf{C}:|z|=1\}, \mathbf{D}=\{w \in \mathbf{C}:|z|<1\}$ and $\mathbf{E}=\{z \in \mathbf{C}:|z|>1\}$ for the unit circle, the open unit disk and the exterior of the unit circle. Finally, let us remark that as for quadratures on $T, L$-polynomials play the same role as the usual polynomials when quadratures for integrals (1) over an interval are considered. This fact is basically motivated by the following

Theorem 1 ([27, p. 39]) Let $C$ be an arbitrary Jordan curve of the finite z-plane. Then, any function $f(z)$ continuous on $C$ can be uniformly approximated on $C$ by the sum of a polynomial in $z$ and a polynomial in $\bar{z}$.

As an immediate consequence, we have

Corollary 1 If $f$ is any continuous function on $\mathbf{T}$, then $f$ can be uniformly approximated on $\mathrm{T}$ by L-polynomials.

\section{Preliminary results}

Let $\mathbf{X}=\left\{x_{j, n}\right\}, n \in \mathbf{N}, j=1,2, \ldots, n$ be a triangular table of nodes, $\mathbf{X} \subset \mathbf{T}$. In order to construct quadrature formulas not involving values of derivatives, we shall assume that $x_{j, n} \neq x_{k, n}$ if $j \neq k$. Thus, for a given integral $I_{\mu}\{f\}$ on the unit circle (2), where $\mu$ is always allowed to be a complex measure, we shall be concerned with its estimation by using a formula of the kind,

$$
I_{n}\{f\}=\sum_{j=1}^{n} A_{j, n} f\left(x_{j, n}\right)
$$


such that $I_{\mu}\{f\}=I_{n}\{f\}$ for any $f \in \mathcal{R}_{n}$, which, as will be established later on, represents a certain subspace of $\Delta$.

Let $G$ be domain of the complex $z$-plane (i.e., a closed and connected point set), which contains the unit circle $\mathrm{T}$ but not the origin. Let $\Gamma$ be the boundary of $G$ which is assumed to be a union of Jordan curves and let $f(z)$ be a function which is analytic in $G$. Then, from Cauchy's Theorem

$$
\frac{1}{2 \pi i} \int_{\Gamma} \frac{z_{0}+z}{z_{0}-z} f(z) d z=-2 z_{0} f\left(z_{0}\right)
$$

whenever $z_{0}$ is in the interior of $G$. Now, setting $g(z)=-f(z) /(2 z)$ (which is also analytic in $G$ ), it follows that

$$
f\left(z_{0}\right)=\frac{1}{2 \pi i} \int_{\Gamma} \frac{z_{0}+z}{z_{0}-z} g(z) d z .
$$

From (5) and Fubini's Theorem, the following holds,

$$
I_{\mu}\{f\}=\frac{1}{2 \pi i} \int_{\Gamma} F_{\mu}(z) g(z) d z
$$

where $F_{\mu}(z)=\int_{-\pi}^{\pi} \frac{e^{i \theta}+z}{e^{i \theta}-z} d \mu(\theta)$ represents the well-known Herglotz-Reisz transform of the measure $\mu$, which is an analytic function outside $\mathbf{T}$.

Let $f_{n}(z)$ denote a certain rational approximant to $F_{\mu}(z)$ with $n$ distinct poles $x_{j, n}$, $j=1, \ldots, n$ on $\mathrm{T}$. That is,

$$
f_{n}(z)=Q_{n}(z) / P_{n}(z) ; \quad \operatorname{deg}\left(Q_{n}\right) \leq \operatorname{deg}\left(P_{n}\right), \quad P_{n}(z)=\prod_{j=1}^{n}\left(z-x_{j, n}\right)
$$

For such rational functions, one has the decomposition,

$$
f_{n}(z)=\lambda_{n}+\sum_{j=1}^{n} A_{j, n} \frac{x_{j, n}+z}{x_{j, n}-z}
$$

and it can be written (again by (5)) as

$$
\frac{1}{2 \pi i} \int_{\Gamma} f_{n}(z) g(z) d z=\frac{1}{2 \pi i} \sum_{j=1}^{n} A_{j, n} \int_{\Gamma} \frac{x_{j, n}+z}{x_{j, n}-z} g(z) d z=\sum_{j=1}^{n} A_{j, n} f\left(x_{j, n}\right)=I_{n}\{f\} .
$$

In other words, when replacing in (6) $F_{\mu}(z)$ by a certain rational approximation, say $f_{n}(z)$, a quadrature formula $I_{n}\{f\}$ of the desired form (3) is obtained.

In order to provide an explicit representation of coefficients $\left\{A_{j, n}\right\}$ in such a formula, we can write

$$
f_{n}(z)=\frac{Q_{n}(z)}{P_{n}(z)}=\frac{a_{0} z^{n}+a_{1} z^{n-1}+\cdots+a_{n}}{z^{n}+b_{1} z^{n-1}+\cdots+b_{n}}=a_{0}+\frac{\alpha_{1} z^{n-1}+\cdots+\alpha_{n}}{z^{n}+b_{1} z^{n-1}+\cdots+b_{n}}
$$


with $\alpha_{i}=a_{i}-a_{0} b_{i} ; i=1,2, \ldots, n$. Thus, $f_{n}(z)=a_{0}+R_{n-1}(z) / P_{n}(z)$, where $R_{n-1} \in \Pi_{n-1}$. Setting, (by simple partial fraction decomposition)

$$
\frac{R_{n-1}(z)}{P_{n}(z)}=\sum_{j=1}^{n} \frac{B_{j}}{z-x_{j, n}}, \quad B_{j}=\frac{R_{n-1}\left(x_{j, n}\right)}{P_{n}^{\prime}\left(x_{j, n}\right)}, \quad j=1, \ldots, n
$$

yields

$$
f_{n}(z)=\lambda_{n}+\sum_{j=1}^{n} A_{j, n} \frac{x_{j, n}+z}{x_{j, n}-z},
$$

where $\lambda_{n}=a_{0}-\sum_{j=1}^{n} B_{j} / 2 x_{j, n}$ and

$$
A_{j, n}=-B_{j} / 2 x_{j, n}, \quad j=1, \ldots, n
$$

since

$$
\frac{1}{z-x_{j, n}}=\frac{1}{2 x_{j, n}}\left[\frac{z+x_{j, n}}{z-x_{j, n}}-1\right] .
$$

As for the error in the numerical quadrature (8), from (6) and (8) one immediately gets,

$$
E_{n}\{f\}=I_{\mu}\{f\}-I_{n}\{f\}=-\frac{1}{2 \pi i} \int_{\Gamma}\left(F_{\mu}(z)-f_{n}(z)\right) \frac{f(z)}{2 z} d z
$$

and it follows trivially that:

$$
\left|E_{n}\{f\}\right| \leq \frac{1}{4 \pi} \max \{|f(\zeta) / \zeta|: \zeta \in \Gamma\} \int_{\Gamma}\left|F_{\mu}(z)-f_{n}(z)\right||d z| .
$$

Equation (12) clearly implies that rational approximants $\left\{f_{n}(z)\right\}$, converging locally uniformly on $\mathbf{C}-\mathrm{T}$ to $F_{\mu}(z)$, can be "good candidates" to provide "suitable quadrature formulas".

In the sequel, we shall assume that, in order to compute (2) with $\mu$ a complex measure, the integrals

$$
\mu_{k}=\int_{-\pi}^{\pi} e^{i k \theta} d \mu(\theta)
$$

exist and are known for $k=0, \pm 1, \pm 2, \ldots$ (Actually, it is sufficient to assume that $\int_{-\pi}^{\pi}|d \mu(\theta)| \leq M<+\infty$.) Furthermore, it can be easily checked that $F_{\mu}(z)$ admits the expansion (around $z=0$ and $z=\infty$ ):

$$
\begin{aligned}
L_{0} & =\mu_{0}+2 \sum_{k=1}^{\infty} \mu_{k} z^{k} ; \quad|z|<1 \\
L_{\infty} & =-\mu_{0}-2 \sum_{k=1}^{\infty} \mu_{-k} z^{-k} ; \quad|z|>1 .
\end{aligned}
$$

Untill now, for a fixed $n$, nothing has been said about the approximation of $F_{\mu}(z)$ by $f_{n}(z)$. Thus, let us assume that $f_{n}(z)$ satisfies

$$
L_{0}-f_{n}(z)=\sum_{j=p+1}^{\infty} \mu_{j}^{*} z^{j}=O\left(z^{p+1}\right), \quad p \geq 0
$$




$$
L_{\infty}-f_{n}(z)=\sum_{j=q+1}^{\infty} \mu_{-j}^{*} z^{-j}=O\left(z^{q+1}\right), \quad q \geq 0 .
$$

Now, if we write (from (7))

$$
f_{n}(z)=\lambda_{n}+\sum_{j=1}^{n} A_{j, n}+2 \sum_{k=1}^{\infty}\left(\sum_{j=1}^{n} A_{j, n} / x_{j, n}^{k}\right) z^{k} ; \quad|z| \rightarrow 0
$$

and

$$
f_{n}(z)=\lambda_{n}-\sum_{j=1}^{n} A_{j, n}-2 \sum_{k=1}^{\infty}\left(\sum_{j=1}^{n} A_{j, n} x_{j, n}^{k}\right) z^{-k} ; \quad|z| \rightarrow \infty,
$$

then, from (15) and (16), one first gets

$$
\lambda_{n}+\sum_{j=1}^{n} A_{j, n}=\mu_{0} \quad \text { and } \quad \lambda_{n}-\sum_{j=1}^{n} A_{j, n}^{n}=-\mu_{0},
$$

which implies $\lambda_{n}=0$. On one hand, we have from (15)

$$
I_{n}\left\{z^{-k}\right\}=\sum_{j=1}^{n} A_{j, n} / x_{j, n}^{k}=\mu_{k}=I_{\mu}\left\{z^{-k}\right\}, \quad\left(z=e^{i \theta}\right), \quad 0 \leq k \leq p
$$

and, on the other, from (16),

$$
I_{n}\left\{z^{k}\right\}=\sum_{j=1}^{n} A_{j, n} x_{j, n}^{k}=\mu_{-k}=I_{\mu}\left\{z^{k}\right\}, \quad 0 \leq k \leq q .
$$

As a result, the following Proposition has been proved.

Proposition 1 Let $n, p$ and $q$ be nonnegative integers $(n \geq 1)$ and $f_{n}(z)$ a rational function (7) satisfying (15-16). Then, $\lambda_{n}=0$ and the formula (3) with weights given by (10) and as nodes the poles of $f_{n}(z)$, is valid in $\Delta_{-p, q}$, that is, $I_{n}\{f\}=I_{\mu}\{f\}$, $\forall f \in \Delta_{-p, q}$.

We see that when formulas (3) valid in subspaces of $\Delta$ (see Corollary 1) are required, so that conditions (15-16) are satisfied, then this clearly leads to the concept of two-point Padé-type approximant $([12,17])$ (As for two-point Padé approximants see, e.g. $[23,20]$.) Thus, in general, let $L$ and $L^{*}$ be two formal power series of the form

$$
L=\sum_{j=0}^{\infty} c_{j} z^{j}, \quad \text { and } \quad L^{*}=\sum_{j=0}^{\infty} c_{-j}^{*} z^{-j}
$$

$k$ and $n$ nonnegative integers such that $0 \leq k \leq n$. Let $P_{n}(z)$ be an arbitrary polynomial of degree $n$, satisfying $P_{n}(0) \neq 0$. Under these conditions a unique polynomial $Q_{n} \in \Pi_{n}$ exists so that (see [12])

$$
L-Q_{n} / P_{n}=O\left(z^{k}\right) \text { and } L^{*}-Q_{n} / P_{n}=O\left(\left(z^{-1}\right)^{n-k+1}\right)
$$


The rational function $Q_{n} / P_{n}$ is said to be a "two-point Padé-type approximant" (2PTA) of order $(k, n-k+1)$ to the pair $\left(L, L^{*}\right)$. According to our purposes (see (15) and (16)) 2PTA's with $k>0$ will be used on the sequel. This means that we shall deal with 2PTA's to the pair (14) and order $(p+1, q+1)$ with $p+q=n-1$. Such approximant will be denoted, in short, by

$$
(p / n)_{L_{0}, L_{\infty}}=Q_{n}(z) / P_{n}(z) ; \quad 0 \leq p \leq n-1, \quad(q=n-1-p)
$$

Remark 1 A reciprocal result to Proposition 1 also holds. Indeed, let $I_{n}\{f\}=$ $\sum_{j=1}^{n} A_{j, n} f\left(x_{j, n}\right)$ be a quadrature formula with distinct nodes and valid in $\Delta_{-p, q}$ $(p+q=n-1)$. If $P_{n}(z)=\prod_{j=1}^{n}\left(z-x_{j, n}\right)$ is the nodal polynomial, then the rational function given by

$$
\sum_{j=1}^{n} A_{j, n} \frac{x_{j, n}+z}{x_{j, n}-z}
$$

represents a 2PTA to $\left(L_{0}, L_{\infty}\right)$ of order $(p+1, q+1)$ with denominator $P_{n}(z)$. For further references concerning connections between rational funcions and quadrature formulas in a rather general framework, see the paper by Njästad and Thron [24].

Remark 2 In order to give a more compact formula for such 2PTA, it will be convenient to recall (see [5]) that a formula (3) is valid in $\Delta_{-p, q}$ with $p+q=n-1$, if and only if $I_{n}\{f\}=I_{\mu}\left\{R_{p, q}\right\}$, where $R_{p, q}$ is unique function in $\Delta_{-p, q}$ satisfying the interpolation conditions $R_{p, q}\left(x_{j, n}\right)=f\left(x_{j, n}\right), j=1,2, \ldots, n$.

On the other hand, one has also,

Proposition 2 Let us consider $g(x, z)=\frac{x+z}{x-z}$ as a function in the variable $x, z$ being a parameter. Let $R_{p, q}$ be the L-polynomial in $\Delta_{-p, q},(p+q=n-1)$ interpolating $g(x, z)$ at distinct gives nodes $x_{j, n}, j=1, \ldots, n\left(x_{j, n} \neq 0\right)$, then

$$
R_{p, q}(x)=1+\frac{2 z}{x-z}\left(1-\frac{z^{p} P_{n}(x)}{x^{p} P_{n}(z)}\right), \quad P_{n}(z)=\prod_{j=1}^{n}\left(z-x_{j, n}\right)
$$

Proof. Since $x_{j, n} \neq 0$ and $P_{n}\left(x_{j, n}\right)=0, R_{p, q}(x)$ given by (17) clearly fullfils the interpolation conditions. Furthermore, if we write,

$$
R_{p, q}(x)=1+\frac{2 z}{P_{n}(z)} x^{-p}\left[\frac{x^{p} P_{n}(z)-z^{p} P_{n}(x)}{x-z}\right]
$$

we see that the expression in brackets is a polynomial in the variable $x$ of degree $n-1$ at most. Therefore, factor $x^{-p}$ makes the resulting function belong to $\Delta_{-p, q}$ and the proof follows by virtue of the unicity property for such interpolating Laurent polynomials. 
Remark 3 The above result is also valid when not necessarily distinct nodes are used. In that case, Hermite interpolation should be considered.

Thus from Proposition 2 and remarks 2-3, one has

Corollary 2 Let $P_{n}(z)$ be a polynomial of degree $n$ such that $P_{n}(0) \neq 0, p$ and $q$ nonnegative integers with $p+q=n-1$. Then, the 2PTA with denominator $P_{n}$ to $F_{\mu}(z)$ (or equivalently to the pair $\left(L_{0}, L_{\infty}\right)$ ) and order $(p+1, q+1)$ is given by

$$
(p / n)_{\left(L_{0}, L_{\infty}\right)}=\frac{Q_{n}(z)}{P_{n}(x)}=I_{\mu}\left\{1+\frac{2 z}{x-z}\left(1-\frac{z^{p} P_{n}(x)}{x^{p} P_{n}(z)}\right)\right\} .
$$

Furthermore, the following formula holds for the error

$$
F_{\mu}(x)-\frac{Q_{n}(x)}{P_{n}(z)}=\frac{2 z^{p+1}}{P_{n}(z)} \int_{-\pi}^{\pi} \frac{e^{-i p \theta} P_{n}\left(e^{i \theta}\right)}{e^{i \theta}-z} d \mu(\theta) .
$$

It results from (18) that the numerator $Q_{n}$ in this approximant, equals

$$
Q_{n}(z)=I_{\mu}\left\{P_{n}(z)+\frac{2 z}{x-z}\left(P_{n}(x)-\frac{z^{p}}{x^{p}} P_{n}(x)\right)\right\}
$$

which yields

$$
Q_{n}(z)=I_{\mu}\left\{\frac{x+z}{x-z}\left(P_{n}(z)-\frac{z^{p}}{x^{p}} P_{n}(x)\right)\right\}+I_{\mu}\left\{\frac{z^{p} P_{n}(x)}{x^{p}}\right\}, \quad 0 \leq p \leq n-1 .
$$

Finally, the following integral expression for coefficients $A_{j, n}$ will be used in Section 4.

Proposition 3 If $I_{n}\{f\}=\sum_{j=1}^{n} A_{j, n} f\left(x_{j, n}\right)$ is a quadrature formula valid in $\Delta_{-p, q}$, $(p+q=n-1)$, then

$$
A_{j, n}=x_{j, n}^{p} I_{\mu}\left\{\frac{z^{-p} P_{n}(z)}{\left(z-x_{j, n}\right) P_{n}^{\prime}\left(x_{j, n}\right)}\right\}, \quad j=1, \ldots, n
$$

where, as usual, $P_{n}(z)=\prod_{j=1}^{n}\left(z-x_{j, n}\right)$.

Proof. Let $R_{p, q}(z)$ be the interpolating $L$-polynomial in $\Delta_{-p, q}$ satisfying $R_{p, q}\left(x_{j, n}\right)=$ $f\left(x_{j, n}\right), j=1, \ldots, n, f$ being a given function on $\mathbf{T}$. Set

$$
L_{j}(z)=x_{j, n}^{p} z^{-p} \frac{P_{n}(z)}{\left(z-x_{j, n}\right) P_{n}^{\prime}\left(x_{j, n}\right)}, \quad j=1, \ldots, n .
$$

Then clearly $L_{j} \in \Delta_{-p, q}$ and $L_{j}\left(x_{k, n}\right)=\delta_{j k} ; 1 \leq j, k \leq n$. Therefore,

$$
R_{p, q}(z)=\sum_{j=1}^{n} L_{j}(x) f\left(x_{j, n}\right)
$$

Now, since $I_{n}\{f\}=I_{\mu}\left\{R_{p, q}\right\}$, the proof follows.

Untill now our starting point has been a triangular table $\mathrm{X}$ of distinct nodes on $\mathbf{T}$ such that for each fixed $n$ a formula (3) valid in $\Delta_{-p, q}(p+q=n-1)$ can be constructed. In the next sections we are concerned with choices of nodes $\left\{x_{j, n}\right\}$ which will give rise to easy computations and which result in sequences of quadrature formulas that are convergent for a class of functions as large as possible. 


\section{Positive measures: Szegö formulas}

As an illustration, we consider in this section the usual case of a finite positive Borel measure $\mu$. By introducing the inner product,

$$
\langle f, g\rangle_{\mu}=\int_{-\pi}^{\pi} f\left(e^{i \theta}\right) \overline{g\left(e^{i \theta}\right)} d \mu(\theta)
$$

a monic sequence of polynomials $\left\{\rho_{n}\right\},\left(\operatorname{deg}\left(\rho_{n}\right)=n\right)$ can be uniquely determined so that $\left\langle\rho_{n}, z^{m}\right\rangle_{\mu}=0$ for $0 \leq m \leq n-1$ and $\left\langle\rho_{n}, \rho_{n}\right\rangle_{\mu}>0$. $\left\{\rho_{n}\right\}$ is called the sequence of Szegö polynomials with respect to the measure $\mu$. Such polynomials have been extensively studied. (See e.g. [21] for a survey and references found there.) Furthermore, as is well known [14, 19], the zeros of the Szegö polynomials all lie inside the unit disc D. Hence, such zeros can not be used as nodes in our quadratures. In order to overcome such drawback, W.B. Jones et al. introduced in [21] certain polynomials with orthogonality properties w.r.t. $\mu$ whose zeros lie on $\mathbf{T}$ and are distinct. Indeed, set $\rho_{n}^{*}(z)=z^{n} \overline{\rho_{n}(1 / \bar{z})}$ and define $X_{n}(z)=\rho_{n}(x)+\omega_{n} \rho_{n}^{*}(z)$ with $\omega_{n} \in \mathbf{T}$, then the following holds (see Th. 6.1 and Th. 6.2 in [21].

Proposition 4 Let $\left\{\omega_{n}\right\}$ be a given sequence of complex numbers satisfying $\left|\omega_{n}\right|=1$ for $n \geq 1$ and let $X_{n}(z)$ be as defined above. Then:

(i) $\left\langle X_{n}, 1\right\rangle_{\mu} \neq 0,\left\langle X_{n}, z^{m}\right\rangle_{\mu}=0,1 \leq m \leq n-1$, and $\left\langle X_{n}, z^{n}\right\rangle_{\mu} \neq 0$

(ii) The $n$ zeros of $X_{n}(z)$ are simple and lie on the unit circle $\mathbf{T}$.

(Polynomials satisfying conditions in (i) are called para-orthogonal.)

For a fixed sequence $\left\{\omega_{n}\right\} \subset \mathrm{T}$, let $x_{j, n}, j=1, \ldots, n$, be the zeros of $X_{n}(z)$, $n=1,2, \ldots$. We now investigate, from our approach, the corresponding quadrature formulas based on such nodes or equivalently the 2PTA's with denominator $X_{n}(z)$. Thus, set

$$
R_{n}^{p}(z)=Q_{n}^{p}(z) / X_{n}(z)=(p / n)_{L_{0}, L_{\infty}}, \quad 0 \leq p \leq n-1 .
$$

Formula (19) and orthogonality properties for $X_{n}(z)$ allow one to show easily that, independently of $p$,

$$
F_{\mu}(z)-R_{n}^{p}(z)=O\left(z^{n}\right) \quad \text { and } \quad F_{\mu}(z)-R_{n}^{p}(z)=O\left((1 / z)^{n}\right) .
$$

From (23) and Proposition 1, we have

Corollary 3 Let $\mu$ be a positive measure on $[-\pi, \pi]$. Let $\left\{x_{j, n}\right\}_{j=1}^{n}$ be the zeros of $X_{n}(z)$ as defined above and $I_{n}\{f\}=\sum_{j=1}^{n} A_{j, n} f\left(x_{j, n}\right)$ the corresponding quadrature formula valid in $\Delta_{-p, q},(p+q=n-1)$. Then, it holds

$$
I_{n}\{f\}=I_{\mu}\{f\}, \quad \forall f \in \Delta_{1-n, n-1} .
$$

Thus, we see from (24) that the so-called Szegö formulas arise, [21]. As for the weights $A_{j, n}$ in those formulas, from (21) and taking $p=0$ (recall that $p$ can be any integer with $0 \leq p \leq n-1)$, it follows that

$$
A_{j, n}=I_{\mu}\left\{\frac{X_{n}(z)}{\left(z-x_{j, n}\right) X_{n}^{\prime}\left(x_{j, n}\right)}\right\}=I_{\mu}\left\{\left|\frac{X_{n}(z)}{\left(z-x_{j, n}\right) X_{n}^{\prime}\left(x_{j, n}\right)}\right|^{2}\right\}>0 .
$$

The last equality in (25) is a consequence of the orthogonality properties for $X_{n}(z)$. (For more details, see [21, pp. 134-135].) 
Remark 4 For the numerator $Q_{n}^{p}(z)$ of the 2PTA $R_{n}^{p}(z)$, from (20) and Proposition 4, (i) (take into account that $I_{\mu}\left\{z^{p} x^{-p} X_{n}(x)\right\}=z^{p}\left\langle X_{n}, x^{p}\right\rangle_{\mu}=0,1 \leq p \leq n-1$ with $\left.x=e^{i \theta}\right)$, the following holds

$$
Q_{n}^{p}(z)=I_{\mu}\left\{\frac{x+z}{x-z}\left(X_{n}(z)-\frac{z^{p}}{x^{p}} X_{n}(x)\right)\right\}, \quad 1 \leq p \leq n-1 .
$$

(Compare with relation (5.6) in [21, p. 127].) Furthermore, if we take $p=0$ in (20), we can also write

$$
Q_{n}^{p}(z)=I_{\mu}\left\{\frac{x+z}{x-z}\left(X_{n}(z)-X_{n}(x)\right)\right\}+\omega_{n}\left\langle z^{n}, \rho_{n}\right\rangle_{\mu} .
$$

Thus, by taking $\varphi_{n}(z)$ as the corresponding orthonormal polynomials of degree $n$ and with leading coefficient $\alpha_{n}>0$, then

$$
Q_{n}^{p}(z)-\frac{\omega_{n}}{\alpha_{n}}=I_{\mu}\left\{\frac{x+z}{x-z}\left(X_{n}(z)-X_{n}(x)\right)\right\}
$$

where $X_{n}(z)=\varphi_{n}(z)+\omega_{n} \varphi_{n}^{*}(z)$ and $\varphi_{n}(z)$ orthonormal.

To end this Section, a result concerning convergence is stated.

Theorem 2 (Convergence of Szegö formulas) Let $\left\{\omega_{n}\right\}$ be a given sequence of complex numbers satisfying $\left|\omega_{n}\right|=1$, for $n \geq 1$. Let $I_{n}\{f\}$ be the sequence of quadratures (Szegó formulas) based on the zeros of $X_{n}(z)=\rho_{n}(z)+\omega_{n} \rho_{n}^{*}(z), n=$ $1,2, \ldots$ (The $\left\{\rho_{n}\right\}$ are the monic Szegô polynomials.) Then, $\lim _{n \rightarrow \infty} I_{n}\{f\}=I_{\mu}\{f\}$ for any integrable function $f$ on $\mathbf{T}$ w.r.t. the measure $\mu$.

Proof. By (11) and Th. 7.3 in [21, pp. 135-136] convergence is first guaranted for $f$ being analytic in D. Corollary 1 and (25) allow to extend convergence to the class of the continuous functions on $\mathrm{T}$. Finally, paralleling rather closely the arguments given in [10, pp. 127-129], one can show that Theorem 2 is also valid for integrable functions, because of the fact that any sequence of integration rules with positive weights, which converges for all continuous functions, converges for all integrable functions w.r.t. a finite Borel measure $\mu$ on $[-\pi, \pi]$.

Remark 5 For an alternative proof of this Theorem see [5]. However, it should be clear that boundedness (12) and relation (19) are valid only for analytic functions $f$.

\section{Complex measures. Choice of nodes and convergence}

Let $\mu$ be a complex measure on $[-\pi, \pi]$, so that nothing, can be said in general, about the location of the zeros of the corresponding Szegö polynomials w.r.t. an inner product $\langle\cdot, \cdot\rangle_{\mu}(22)$ which is not Hermitian. As a result, for a given sequence of 
para-orthogonal polynomials (in case they exist), their zeros do not necessarily lie on $\mathbf{T}$ and cannot be used as nodes in a quadrature formula (3). Therefore we describe a method by which easily computable nodes can be selected such that, convergence of the quadrature process is assured. Let us first consider the "natural" selection of equally distributed nodes $\left\{x_{j, n}\right\}$ on $\mathbf{T}$; that is,

$$
P_{n}(z)=\prod_{j=1}^{n}\left(z-x_{j, n}\right)=z^{n}+\omega_{n}, \quad\left|\omega_{n}\right|=1, \quad n=1,2, \ldots
$$

Let $\{p(n)\}_{1}^{\infty}$ and $\{q(n)\}_{1}^{\infty}$ be two arbitrary sequences of nonnegative integers with $p(n)+q(n)=n-1$ and $\lim _{n \rightarrow \infty} \frac{p(n)}{n}=r, 0<r<1$. We consider the sequence of quadrature formulas (3) with nodes given by (26), so that for $n \geq 1, I_{n}\{f\}$ is exact in $\Delta_{-p(n), q(n)}$. One has,

Theorem 3 Let $I_{n}\{f\}$ and $\mu$ be as in the preceding paragraph. Let $f$ be an analytic function in a domain $G$ such that $\mathrm{T} \subset G$ and $0 \notin G$. Then, $\lim _{n \rightarrow \infty} I_{n}\{f\}=I_{\mu}\{f\}$. Furthermore,

$$
\lim _{n \rightarrow \infty}\left|E_{n}\{f\}\right|^{1 / n}=\lim _{n \rightarrow \infty}\left|I_{n}\{f\}-I_{\mu}\{f\}\right|^{1 / n} \leq \gamma<1,
$$

where $\gamma=\max \left\{\gamma_{1}, \gamma_{2}\right\}$ with $\gamma_{1}=\max \left\{|z|^{r}: z \in \Gamma \cap \mathbf{D}\right\}$ and $\gamma_{2}=\max \left\{|z|^{1-r}: z \in\right.$ $\Gamma \cap \mathbf{E}\}, \Gamma$ being the boundary of $G$.

Proof. By (12) it suffices to prove that the sequence of 2PTA's of order $(p(n)+$ $1, q(n)+1)$ with denominators $(26)$ converge locally uniformly to $F_{\mu}(z)$ outside $\mathrm{T}$. Indeed, for any $z \notin T$, (19) yields,

$$
\left|F_{\mu}(z)-\frac{Q_{n}(z)}{P_{n}(z)}\right| \leq \frac{2|z|^{p(n)+1}}{\left|P_{n}(z)\right|} \max _{x \in \mathbf{T}}\left|P_{n}(x)\right| \int_{-\pi}^{\pi} \frac{|d \mu(\theta)|}{\left|e^{i \theta}-z\right|} .
$$

Now, if $z \in \mathbf{D}$ then $\lim _{n \rightarrow \infty}\left|P_{n}(z)\right|^{1 / n}=1$. Therefore, from (28)

$$
\lim _{n \rightarrow \infty}\left|F_{\mu}(z)-Q_{n}(z) / P_{n}(z)\right|^{1 / n} \leq|z|^{r}<1 .
$$

Next, let us assume that $z \in \mathbf{E}$, that is $|z|>1$. Since

$$
\left|P_{n}(z)\right|=\left|z^{n}+\omega_{n}\right|=\left|z^{n}\right|\left|z^{-n}+\bar{\omega}_{n}\right|, \quad\left(\omega_{n} \in \mathrm{T}\right),
$$

then clearly $\lim _{n \rightarrow \infty}\left|P_{n}(z)\right|^{1 / n}=|z|$ for any $z \in \mathbf{E}$, which implies

$$
\lim _{n \rightarrow \infty}\left|F_{\mu}(z)-Q_{n}(z) / P_{n}(z)\right|^{1 / n} \leq|z|^{r-1}<1 .
$$

Hence, by (29), (30) and (11) the proof follows.

In order to study other selections of nodes $\left\{x_{j, n}\right\} \subset \mathbf{T}$, it is helpful to recall that for integrals (1), interpolatory quadrature formulas based on zeros of orthogonal polynomials w.r.t. a positive measure on $[a, b]$ have been largely considered in recent 
years (see e.g. [2]). Accordingly, for a given positive measure $\alpha$ on $[-\pi, \pi]$, we let $\left\{X_{n}^{\alpha}\right\}$ be a sequence of para-orthogonal polynomials, that is

$$
X_{n}^{\alpha}(z)=\phi_{n}(x, \alpha)+\omega_{n} \phi_{n}^{*}(z, \alpha)
$$

$\left\{\phi_{n}(z, \alpha)\right\}$ being the monic Szegö polynomials w.r.t. $\alpha$. Thus quadrature formulas (3) based on the zeros of $X_{n}^{\alpha}(z)$ to estimate $I_{\mu}\{f\}$ could provide good results. Immediately, a question arises: Which measure $\alpha$ should be chosen?

By taking $d \alpha(\theta)=d \theta /(2 \pi)$ (Lebesgue measure), then $\phi_{n}(z, \alpha)=z^{n}$ and $X_{n}^{\alpha}(z)=$ $z^{n}+\omega_{n}$. Thus, the case of equally distributed nodes on $\mathbf{T}$ is now contemplated. We can also think of measures induced by the Poisson kernel, that is

$$
d \alpha(\theta)=\frac{1}{2 \pi} \frac{1-|r|^{2}}{|z-r|^{2}} d \theta, \quad z=e^{i \theta} \quad \text { and } \quad r \in \mathrm{D} .
$$

(Observe that when $r=0$, the Lebesgue measure is recovered.) We now find $\phi_{0}(z, \alpha)=\phi_{0}^{*}(z, \alpha)=1, \phi_{n}(z, \alpha)=z^{n}-r z^{n-1}, \phi_{n}^{*}(z, \alpha)=1-\bar{r} z, n \geq 1$ and for the para-orthogonal polynomials [26],

$$
X_{n}^{\alpha}(z)=z^{n}-r z^{n-1}-\omega_{n} \bar{r} z+\omega_{n} .
$$

From (32) $z=1$ is a zero iff $r \in \mathbf{R}$ and $\omega_{n}=-1$, and $z=-1$ iff $\omega_{n}=(-1)^{n-1}$. When, for simplicity, we choose $\omega_{n}=1, n \geq 1$, all other zeros come in conjugate pairs whenever $r$ is real, $0<r<1$. (Take into account that the more general case $r \in \mathbf{D}$ can be easily reduced to this one.) In any case, for large $n$-values, the zeros of (32) must be computed numerically. As for the convergence of the corresponding sequence of quadrature formulas based on polynomials (32), one has: for any given sequence $\left\{\omega_{n}\right\} \subset \mathrm{T}$ and $z \in \mathbf{D}$,

$$
\lim _{n \rightarrow \infty}\left|X_{n}^{\alpha}(z)\right|^{1 / n}=1 .
$$

Furthermore, $X_{n}^{\alpha}(z)=\omega_{n} z^{n} T_{n}^{\alpha}(1 / z)$, where $T_{n}^{\alpha}(z)=\phi_{n}(z, \alpha)+\bar{\omega}_{n} \phi_{n}^{*}(z, \alpha)$. Hence, for any $z \in \mathbf{E}$, it results

$$
\lim _{n \rightarrow \infty}\left|X_{n}^{\alpha}(z)\right|^{1 / n}=|z| \text {. }
$$

Thus, from (33-34), Theorem 3 remains still valid when replacing polynomials (26) $(r=0)$ by polynomials $(32)(r \in \mathbf{D})$. Hence, from the point of view of the rate of convergence for the sequence $I_{n}\{f\}$, measures (31) do not seem to provide relevant contributions. Certainly the problem of choosing the most appropriate measure is an open question and much more work could be done on this in the future. To end, the extension of Theorem 3 to the class of continuous functions on $\mathrm{T}$ is studied. By Corollary 1 , this extension would be a simple matter, provided that the sequence $S_{n}=\sum_{j=1}^{m}\left|A_{j, n}\right|$ is bounded. As usual $A_{j, n}$ are the weights in formula (3); that is, (recall (21)),

$$
A_{j, n}=x_{j, n}^{p(n)} \int_{-\pi}^{\pi} \frac{z^{-p(n)} P_{n}(z)}{\left(z-x_{j, n}\right) P_{n}^{\prime}\left(x_{j, n}\right)} d \mu(\theta), \quad z=e^{i \theta} .
$$


On the other hand, if $P_{n}$ is para-orthogonal w.r.t. a positive measure $\alpha$ supported in $[-\pi, \pi]$, the weights $\lambda_{j, n}$ in the Szegö formula (Section 3) are given by (25):

$$
\lambda_{j, n}=\int_{-\pi}^{\pi}\left|\frac{P_{n}(z)}{\left(z-x_{j, n}\right) P_{n}^{\prime}\left(x_{j, n}\right)}\right|^{2} d \alpha(\theta), \quad z=e^{i \theta} ; \quad j=1,2, \ldots, n .
$$

The measure $\alpha$ is also assumed to be normalized, i.e. $\int d \alpha(\theta)=1$. So, we have

$$
\sum_{j=1}^{n} \lambda_{j, n}=1
$$

Now, let us assume that $d \alpha(\theta)=\alpha^{\prime}(\theta) d \theta, \alpha^{\prime}(\theta)>0$, a.e. in $[-\pi, \pi], d \mu(\theta)=w(\theta) d \theta$ and,

$$
\int_{-\pi}^{\pi} \frac{|w(\theta)|^{2}}{\alpha^{\prime}(\theta)} d \theta=K<+\infty
$$

Then, making use of the Cauchy-Schwartz inequality, we can write $\left(z=e^{i \theta}\right)$ :

$$
\begin{aligned}
\left|A_{j, n}\right| & =\left|\int_{-\pi}^{\pi}\left[\frac{P_{n}(z)}{\left(z-x_{j, n}\right) P_{n}^{\prime}\left(x_{j, n}\right)} \sqrt{\alpha^{\prime}(\theta)}\right] \frac{w(\theta) z^{-p(n)}}{\sqrt{\alpha^{\prime}(\theta)}} d \theta\right| \leq \\
& \leq\left\{\int_{-\pi}^{\pi}\left|\frac{P_{n}(z)}{\left(z-x_{j, n}\right) P_{n}^{\prime}\left(x_{j, n}\right)}\right|^{2} \alpha^{\prime}(\theta) d \theta\right\}^{1 / 2}\left\{\int_{-\pi}^{\pi} \frac{|w(\theta)|^{2}}{\alpha^{\prime}(\theta)} d \theta\right\}^{1 / 2}=K_{1} \lambda_{j, n}^{1 / 2},
\end{aligned}
$$

with $K_{1}=K^{1 / 2}$. Therefore, by using again the Cauchy-Schwartz inequality, we get

$$
S_{n}=\sum_{j=1}^{n}\left|A_{j, n}\right| \leq K_{1} \sum_{j=1}^{n}\left[\lambda_{j, n}\right]^{1 / 2} \leq K_{1} \sqrt{n}\left\{\sum_{j=1}^{n} \lambda_{j, n}\right\}^{1 / 2}=K_{1} \sqrt{n} .
$$

Remark 6 Under the above conditions, for a positive measure $\alpha$, we have in general the inequality $\sum_{j=1}^{n}\left[\lambda_{j, n}\right]^{1 / 2} \leq \sqrt{n}$ which turns out to be an equality when taking the Lebesgue measure, i.e. $d \alpha(\theta)=d \theta / 2 \pi$, because then $\lambda_{j, n}=1 / n, j=1,2, \ldots, n$. (See, [26] and [8].)

As is seen from (37), we cannot in general conclude that the sequence $\left\{S_{n}\right\}$ is bounded; thus additional assumptions on the function $f$ are required. For a continuous function $f$ on $\mathbf{T}$, one can write

$$
f\left(e^{i \theta}\right)=f_{1}(\theta)+i f_{2}(\theta), \quad \theta \in[-\pi, \pi]
$$

where $f_{1}(\theta)=\operatorname{Re}\left(f\left(e^{i \theta}\right)\right)$ and $f_{2}(\theta)=\operatorname{Im}\left(f\left(e^{i \theta}\right)\right)$ are $2 \pi$-periodic continuous functions on $[-\pi, \pi]$. Let us introduce the modulus of continuity for the function $f$,

$$
\omega(f, \delta)=\max \left\{\left|f\left(e^{i s}\right)-f\left(e^{i t}\right)\right|:|s-t|<\delta\right\}, \quad-\pi<s, t<\pi .
$$


Let $C_{2 \pi}$ stand for the space of continuous $2 \pi$-periodic functions with supremum norm. For $g \in C_{2 \pi}$, we write

$$
\gamma_{n}(g)=\min _{a_{k}, b_{k}} \max _{\theta}\left|g(\theta)-\sum_{k=1}^{n}\left(a_{k} \cos k \theta+b_{k} \sin k \theta\right)\right|, \quad a_{k}, b_{k} \in \mathbf{R} .
$$

For our purposes, the following result will be needed,

Theorem 4 (Jackson's Theorem III, [9] p. 144)

$$
\forall g \in C_{2 \pi}: \quad \gamma_{n}(g) \leq \omega\left(g, \frac{\pi}{n+1}\right) .
$$

Now, we are in a situation to prove the following:

Theorem 5 If $f$ is a continuous function on $\mathbf{T}$, then there exists $R_{n} \in \Delta_{-n, n}$ such that,

$$
\max _{z \in \mathbf{T}}\left|f(z)-R_{n}(z)\right| \leq 2 \omega\left(f, \frac{\pi}{n+1}\right) .
$$

Proof. Let $R_{n, j}(\theta), j=1,2$ be the real trigonometric polynomials satisfying

$$
\gamma_{n}\left(f_{1}\right)=\max _{\theta}\left|f_{1}(\theta)-R_{n, 1}(\theta)\right| \quad \text { and } \quad \gamma_{n}\left(f_{2}\right)=\max _{\theta}\left|f_{2}(\theta)-R_{n, 2}(\theta)\right|
$$

with $f_{j}(\theta), j=1,2$ given by (38). Set $R_{n, 1}(\theta)=\operatorname{Re} R_{n}$ and $R_{n, 2}(\theta)=\operatorname{Im} R_{n}$, i.e.,

$$
R_{n}(z)=R_{n, 1}(\theta)+i R_{n, 2}(\theta) ; \quad z=e^{i \theta} .
$$

Then, $R_{n} \in \Delta_{-n, n}$. Furthermore, since

$$
\left|f_{j}(s)-f_{j}(t)\right| \leq\left|f\left(e^{i s}\right)-f\left(e^{i t}\right)\right|, \quad j=1,2
$$

it follows that,

$$
\omega\left(f_{j}, \delta\right) \leq \omega(f, \delta) ; \quad j=1,2 .
$$

Thus, by Theorem 4 and (38),

$$
\max _{z \in \mathbf{T}}\left|f(z)-R_{n}(z)\right| \leq \max _{\theta}\left|f_{1}(\theta)-R_{n, 1}(\theta)\right|+\max _{\theta}\left|f_{2}(\theta)-R_{n, 2}(\theta)\right| \leq 2 \omega\left(f, \frac{\pi}{n+1}\right)
$$

It follows that, if $f$ is a continuous function on $\mathbf{T}$ with $\omega(f, \delta)=O\left(\delta^{p}\right)$ with $p>1 / 2$, then by Theorem 5 and (37), constants $A, B$ and $C$ indenpendent of $n$ exist so that for sufficiently large $n$, one gets

$$
\left|I_{n}\{f\}-I_{\mu}\{f\}\right| \leq \frac{A+B \sqrt{n}}{(C+n)^{p}} .
$$

In other words, the following Theorem has been proved. 
Theorem 6 Let $f$ be a continuous function on T with modulus of continuity $\omega(f, \delta)=$ $O\left(\delta^{p}\right)(p>1 / 2)$ and, for $n=1,2, \ldots$, let $\left\{x_{j, n}\right\}$ be the zeros of the para-orthogonal polynomials $X_{n}^{\alpha}(z)$ w.r.t. a positive measure $\alpha$ supported in $[-\pi, \pi]$. Then, if the measure $\mu$ satisfies (36), the sequence of quadrature formulas for $\mu$ with nodes $\left\{x_{j, n}\right\}$ converges to $I_{\mu}\{f\}$.

Corollary 4 Under the conditions of Theorem 6 , the sequence $I_{n}\{f\}$ of quadrature formulas (3) with equally distributed nodes on $\mathrm{T}$, converges to $I_{\mu}\{f\}$.

Remark 7 Note that Theorem 6 holds for any function $f$ satisfying a Lipschitz condition of the form:

$$
\left|f\left(z_{1}\right)-f\left(z_{2}\right)\right| \leq \lambda\left|z_{1}-z_{2}\right|^{p}, \quad(p>1 / 2) ; \quad z_{1}, z_{2} \in \mathbf{T} .
$$

\section{References}

1. D. Barrios, G. López, and E. Torrano. Location of zeros and asymptotics of polynomials satisfying three-term recurrence relations. Math. Sbornik, 1993. To appear.

2. T. Bloom, D.S. Lubinski, and H. Stahl. Interpolatory integration rules and orthogonal polynomials with varying weights. Numerical Algorithms, 3:55-66, 1992.

3. A. Bultheel. Algorithms to compute the reflection coefficients of digital filters. In L. Collatz, G. Meinardus, and H. Werner, editors, Numerische Methoden der Approximationstheorie 7 , volume 67 of ISNM, pages 33-50. Birkhäuser Verlag, 1984.

4. A. Bultheel, P. González-Vera, E. Hendriksen, and O. Njảstad. A Szegö theory for rational functions. Technical Report TW131, Department of Computer Science, K.U. Leuven, May 1990.

5. A. Bultheel, P. González-Vera, E. Hendriksen, and O. Njästad. Orthogonality and quadrature on the unit circle. In C. Brezinski, L. Gori, and A. Ronveaux, editors, Orthogonal polynomials and their applications, volume 9 of IMACS annals on computing and applied mathematics, pages 205-210, Basel, 1991. J.C. Baltzer AG.

6. A. Bultheel, P. González-Vera, E. Hendriksen, and O. Njảstad. Orthogonal rational functions and quadrature on the unit circle. Numer. Alg., 3:105-116, 1992.

7. A. Bultheel, P. González-Vera, E. Hendriksen, and O. Njảstad. Quadrature formulas on the unit circle based on rational functions. J. Comput. Appl. Math., 1992. To appear.

8. M. Camacho and P. González-Vera. A note on para-orthogonality and biorthogonality. Det Kongelige Norske Videnskaber Selskab, Skrifter 3:1-16, 1992.

9. E.W. Cheney. Introduction to approximation theory. McGraw Hill, 1966.

10. P.J. Davis and P. Rabinowitz. Methods of numerical integration. Academic Press, 2nd edition, 1984.

11. Ph. Delsarte, Y. Genin, Y. Kamp, and P. Van Dooren. Speech modelling and the trigonometric moment problem. Philips J. of Research, 37:277-292, 1982.

12. A. Draux. Approximation de type-Padé en deux points. Technical Report ANO-110, Université des Sciences et Techniques de Lille, Lab. d'analyse numérique et d'optimisation, 1983.

13. W. Gautchi. A survey of Gauss-Christoffel quadrature formulae. In P.L. Butzer and F. Feher, editors, E.B. Christoffel. The influence of his work on mathematical and physical sciences, pages 72-147, Basel, 1081. Birkhäuser Verlag.

14. Ya. Geronimus. Polynomials orthogonal on a circle and their applications, volume 3 of Trans. Math. Monographs, Ser. 1, pages 1-78. AMS, 1954.

15. I. Gohberg, editor. I. Schur methods in operator theory and signal processing, volume 18 of Operator Theory: Advances and Applications. Birkhäuser Verlag, Basel, 1986.

16. P. González-Vera, G. López, R. Orive, and J.C. Santos-Leon. On the convergence of quadrature formulas for complex weight functions. Preprint., 1993. 
17. P. González-Vera and R. Orive. Optimization of two-point Padé-type approximants. J. Comp. Appl. Math., 1993. To appear.

18. P. González-Vera, J.C. Santos-Leon, and O. Njästad. Some numerical results about quadrature formulas on the unit circle. Preprint., 1993.

19. U. Grenander and G. Szegö. Toeplitz forms and their applications. University of California Press, Berkley, 1958.

20. W.B. Jones, O. Njästad, and W.J. Thron. Two-point Padé expansions for a family of analytic functions. J. Comp. Appl. Math., 9:105-124, 1983.

21. W.B. Jones, O. Njästad, and W.J. Thron. Moment theory, orthogonal polynomials, quadrature and continued fractions associated with the unit circle. Bull. London Math. Soc., 21:113$152,1989$.

22. H.J. Landau. Maximum entropy and the moment problem. Bull. Am. Math. Soc. (New Series), 16(1):47-77, 1987.

23. A. Magnus. On the structure of the two-point Padé table. In Analytic theory of continued fractions, volume 932 of Lecture Notes Math., pages 362-372, Berlin, 1982. Springer.

24. O. Njästad and W.J. Thron. Rational functions and quadrature formulas. Analysis, 8:347-366, 1988.

25. J. Nuttal and C.J. Wherry. Gauss integration for complex weight functions. J. Inst. Math. Appl., 21:165-170, 1987.

26. H. Waadeland. A Szegö quadrature formula for the Poisson integral. In Proceedings IMACS'91 (Dublin), pages 12-13, 1991.

27. J. L. Walsh. Interpolation and approximation, volume 20 of AMS Colloquium publications. American Mathematical Society, Providence, Rhode Island, 3rd edition, 1960. First edition 1935. 